Supporting Material for

\title{
Structure and Stability of the $[\mathrm{TCNE}]_{2}{ }^{2-}$ Dimers in
}

\section{Dichloromethane Solution - A Computational Study}

Iñigo Garcia-Yoldi, ${ }^{a}$ Joel S. Miller, ${ }^{b}$ Juan J. Novoa ${ }^{a}$

a Departament de Química Física, Facultat de Química \& CERQT, Parc

Cientìfic, Universitat de Barcelona, Av. Diagonal, 647, 08028-Barcelona (Spain)

${ }^{\mathrm{b}}$ Department of Chemistry, University of Utah, Salt Lake City, Utah 84112-0850 (USA)

\section{Complete list of authors for reference 29:}

Gaussian-03, Revision-C.02, Frisch, M. J.; Trucks, G. W.; Schlegel, H. B.; Scuseria, G. E.; Robb, M. A.; Cheeseman, J. R.; Montgomery, Jr., J. A.; Vreven, T.; Kudin, K. N.; Burant, J. C.; Millam, J. M.; lyengar, S. S.; Tomasi, J.; Barone, V.; Mennucci, B.; Cossi, M.; Scalmani, G.; Rega, N.; Petersson, G. A.; Nakatsuji, H.; Hada, M.; Ehara, M.; Toyota, K.; Fukuda, R.; Hasegawa, J.; Ishida, M.; Nakajima, T.; Honda, Y.; Kitao, O.; Nakai, H.; Klene, M.; Li, X.; Knox, J. E.; Hratchian, H. P.; Cross, J. B.; Bakken, V.; Adamo, C.; Jaramillo, J.; Gomperts, R.; Stratmann, R. E.; Yazyev, O.; Austin, A. J.; Cammi, R.; Pomelli, C.; Ochterski, J. W.; Ayala, P. Y.; Morokuma, K.; Voth, G. A.; Salvador, P.; Dannenberg, J. J.; Zakrzewski, V. G.; Dapprich, S.; Daniels, A. D.; Strain, M. C.; Farkas, O.; Malick, D. K.; Rabuck, A. D.; Raghavachari, K.; Foresman, J. B.; Ortiz, J. V.; Cui, Q.; Baboul, A. G.; Clifford, S.; Cioslowski, J.; Stefanov, B. B.; Liu, G.; Liashenko, A.; Piskorz, P.; Komaromi, I.; Martin, R. L.; Fox, D. J.; Keith, T.; Al-Laham, M. A.; Peng, C. Y.; Nanayakkara, A.; Challacombe, M.; Gill, P. M. W.; Johnson, B.; Chen, W.; Wong, M. W.; Gonzalez, C.; and Pople, J. A.; Gaussian, Inc., Wallingford CT, 2004. 
Table S1. Optimum geometry for the $(\mathrm{TCNE})_{2}\left(\mathrm{CH}_{2} \mathrm{Cl}_{2}\right)_{4}$ triplet computed at the UB3LYP/6-31+g(d) level. The total energy, lowest vibrational frequencies, atomic spin population and atomic charge population are also given.

\begin{tabular}{|c|c|c|c|}
\hline C & 000 & 00 & 0.720878 \\
\hline C & 0.000000 & 0.000000 & \\
\hline C & 226961 & 0.000000 & -0 \\
\hline C & 226961 & 0.000000 & 0.7208 \\
\hline C & 05604 & 1.202292 & \\
\hline C & 005604 & -1.202292 & 46 \\
\hline C & .005604 & 1.202292 & -1.4620 \\
\hline C & 05604 & -1.202292 & \\
\hline C & .221358 & 1.202292 & -1.4620 \\
\hline C & 221358 & -1.202292 & -1.462 \\
\hline$\Omega$ & 221358 & 1.202292 & 1.462 \\
\hline & $\mid 358$ & -1.202292 & 1.4620 \\
\hline $\mathrm{N}$ & .008418 & 2.189825 & $2.091 \varepsilon$ \\
\hline $\mathrm{N}$ & .008418 & -2.189825 & $2.091 \varepsilon$ \\
\hline $\mathrm{N}$ & .008418 & 2.189825 & -2.0918 \\
\hline 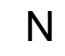 & 18 & -2.1 & -2.09 \\
\hline $\mathrm{N}$ & 44 & 2.1 & -2.09 \\
\hline$N$ & .218544 & -2.1 & -2.0918 \\
\hline  & .218544 & 2.189825 & 2.09 \\
\hline $\mathrm{N}$ & 5.21 & -2 & $2 .($ \\
\hline C & 96 & -4 & 3.5 \\
\hline 1 & 1.706799 & -3.55 & 3.1198 \\
\hline $\mathrm{H}$ & & -3.5 & \\
\hline r & 2.6 & -3.7 & 5.3 \\
\hline $\mathrm{Cl}$ & 2.613792 & -5.7 & 3.060 \\
\hline C & & & \\
\hline 1 & 1.70 & 3.5 & 3.119 \\
\hline $\mathrm{H}$ & 3.520792 & 3.5 & 3.1198 \\
\hline $\mathrm{Cl}$ & & & \\
\hline $\mathrm{Cl}$ & 2.61 & $3.7 \varepsilon$ & $5.30 €$ \\
\hline C & 2.613796 & 4.00 & -3.5170 \\
\hline 4 & & & \\
\hline 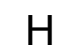 & 3.52 & 3.5 & -3.1 \\
\hline $\mathrm{Cl}$ & 2.61 & 3.782863 & -5.3061 \\
\hline $\mathrm{Cl}$ & 2.61 & & \\
\hline C & 2.613 & -4.00 & -3.51 \\
\hline (11 & 1.70 & -3.5 & -3.11 \\
\hline $\mathrm{H}$ & 3.520792 & -3.555 & -3.11 \\
\hline $\mathrm{Cl}$ & 2.613792 & -5.753123 & $-3.06 c$ \\
\hline & 2.613792 & 0.700 & \\
\hline
\end{tabular}

$\mathrm{E}(\mathrm{UB}+\mathrm{HF}-\mathrm{LYP})=-4734.11673444$

$\left\langle S^{\star *} 2>=2.0179\right.$ 


$\begin{array}{ccccc} & 1 & 2 & 3 & \\ \text { Frequencies -- } & 4.9903 & 9.7513 & & 10.4790 \\ & 4 & 5 & 6 & \\ \text { Frequencies -- } & 11.3948 & 11.8525 & & 14.0486\end{array}$

Mulliken atomic spin densities

\begin{tabular}{|c|c|}
\hline $\mathrm{TCNE}^{-}(1)$ & 0.99 \\
\hline $\operatorname{TCNE}^{-}(2)$ & 0.99 \\
\hline $\mathrm{CH}_{2} \mathrm{Cl}_{2}(1)$ & 0.00 \\
\hline $\mathrm{CH}_{2} \mathrm{Cl}_{2}(2)$ & 0.00 \\
\hline $\mathrm{CH}_{2} \mathrm{Cl}_{2}$ (3) & 0.00 \\
\hline $\mathrm{CH}_{2} \mathrm{Cl}_{2}(4)$ & 0.00 \\
\hline
\end{tabular}

Mulliken atomic charges:

$\begin{array}{ll}\mathrm{TCNE}^{-}(1) & -1.05 \\ \text { TCNE- (2) } & -1.05 \\ & \\ \mathrm{CH}_{2} \mathrm{Cl}_{2}(1) & 0.03 \\ \mathrm{CH}_{2} \mathrm{Cl}_{2}(2) & 0.03 \\ \mathrm{CH}_{2} \mathrm{Cl}_{2}(3) & 0.03 \\ \mathrm{CH}_{2} \mathrm{Cl}_{2}(4) & 0.03\end{array}$


Table S2. Optimum geometry for the $(\mathrm{TCNE})_{2}\left(\mathrm{CH}_{2} \mathrm{Cl}_{2}\right)_{4}$ closed-shell singlet computed at the UB3LYP/6-31+g(d) level. The total energy, lowest vibrational frequencies, atomic spin population and atomic charge population are also given.

$\begin{array}{cccc}6 & 0.000000 & 0.718900 & 1.507302 \\ 6 & 0.000000 & -0.718900 & 1.507302 \\ 6 & 0.000000 & -0.718900 & -1.507198 \\ 6 & 0.000000 & 0.718900 & -1.507198 \\ 6 & -1.200534 & 1.455625 & 1.651232 \\ 6 & 1.200534 & 1.455625 & 1.651232 \\ 6 & -1.200534 & -1.455625 & 1.651232 \\ 6 & 1.200534 & -1.455625 & 1.651232 \\ 6 & -1.200554 & -1.455608 & -1.651128 \\ 6 & 1.200554 & -1.455608 & -1.651128 \\ 6 & -1.200554 & 1.455608 & -1.651128 \\ 6 & 1.200554 & 1.455608 & -1.651128 \\ 7 & -2.187685 & 2.065799 & 1.800968 \\ 7 & 2.187685 & 2.065799 & 1.800968 \\ 7 & -2.187685 & -2.065799 & 1.800968 \\ 7 & 2.187685 & -2.065799 & 1.800968 \\ 7 & -2.187713 & -2.065769 & -1.800864 \\ 7 & 2.187713 & -2.065769 & -1.800864 \\ 7 & -2.187713 & 2.065769 & -1.800864 \\ 7 & 2.187713 & 2.065769 & -1.800864 \\ 6 & 4.437630 & 3.753701 & 0.000000 \\ 1 & 3.967835 & 3.356301 & 0.896361 \\ 1 & 3.967835 & 3.356301 & -0.896257 \\ 17 & 4.247312 & 5.548338 & 0.000000 \\ 17 & 6.175869 & 3.268453 & 0.000000 \\ 6 & -4.437630 & 3.753701 & 0.000000 \\ 1 & -3.967835 & 3.356301 & 0.896361 \\ 1 & -3.967835 & 3.356301 & -0.896257 \\ 17 & -6.175869 & 3.268453 & 0.000000 \\ 17 & -4.247312 & 5.548338 & 0.000000 \\ 6 & -4.437630 & -3.753701 & 0.000000 \\ 1 & -3.967835 & -3.356301 & 0.896361 \\ 1 & -3.967835 & -3.356301 & -0.896257 \\ 17 & -4.247312 & -5.548338 & 0.000000 \\ 17 & -6.175869 & -3.268453 & 0.000000 \\ 6 & 4.437630 & -3.753701 & 0.000000 \\ 1 & 3.967835 & -3.356301 & 0.896361 \\ 1 & 3.967835 & -3.356301 & -0.896257 \\ 17 & 6.175869 & -3.268453 & 0.000000 \\ 17 & 4.247312 & -5.548338 & 0.000000 \\ & & & \\ \mathrm{E}(\mathrm{UB}+\mathrm{HF}-\mathrm{LYP})= & -4734.10233492 \\ <\mathrm{S}^{\star *} 2> & =0.0000 & & \\ & & & \\ & & \end{array}$




$\begin{array}{ccccc} & 1 & 2 & 3 & \\ \text { Frequencies -- } & 9.0626 & 9.4530 & & 9.8017 \\ \text { Frequencies -- } & 9.8623 & 5 & 6 & \\ \text { F } & 9.86 .5705 & & 17.9913\end{array}$

Mulliken atomic spin densities

\begin{tabular}{|c|c|}
\hline TCNE$^{-}$(1) & 0.00 \\
\hline TCNE$^{-}(2)$ & 0.00 \\
\hline $\mathrm{CH}_{2} \mathrm{Cl}_{2}(1)$ & 0.00 \\
\hline $\mathrm{CH}_{2} \mathrm{Cl}_{2}(2)$ & 0.00 \\
\hline $\mathrm{CH}_{2} \mathrm{Cl}_{2}$ (3) & 0.00 \\
\hline $\mathrm{CH}_{2} \mathrm{Cl}_{2}(4)$ & 0.00 \\
\hline
\end{tabular}

Mulliken atomic charges:

$\begin{array}{ll}\text { TCNE- (1) } & -1.06 \\ \text { TCNE- (2) } & -1.06 \\ & \\ \mathrm{CH}_{2} \mathrm{Cl}_{2}(1) & 0.03 \\ \mathrm{CH}_{2} \mathrm{Cl}_{2}(2) & 0.03 \\ \mathrm{CH}_{2} \mathrm{Cl}_{2}(3) & 0.03 \\ \mathrm{CH}_{2} \mathrm{Cl}_{2}(4) & 0.03\end{array}$


Table S3. Optimum geometry for the (TCNE $)_{2}\left(\mathrm{CH}_{2} \mathrm{Cl}_{2}\right)_{4}$ open-shell singlet computed at the UB3LYP/6-31+g(d) level. The total energy, lowest vibrational frequencies, atomic spin population and atomic charge population are also given.

$\begin{array}{rrrr}\mathrm{C} & 0.000000 & 0.000000 & 0.720878 \\ \mathrm{C} & 0.000000 & 0.000000 & -0.720878 \\ \mathrm{C} & 5.226961 & 0.000000 & -0.720878 \\ \mathrm{C} & 5.226961 & 0.000000 & 0.720878 \\ \mathrm{C} & 0.005604 & 1.202292 & 1.462046 \\ \mathrm{C} & 0.005604 & -1.202292 & 1.462046 \\ \mathrm{C} & 0.005604 & 1.202292 & -1.462046 \\ \mathrm{C} & 0.005604 & -1.202292 & -1.462046 \\ \mathrm{C} & 5.221358 & 1.202292 & -1.462046 \\ \mathrm{C} & 5.221358 & -1.202292 & -1.462046 \\ \mathrm{C} & 5.221358 & 1.202292 & 1.462046 \\ \mathrm{C} & 5.221358 & -1.202292 & 1.462046 \\ \mathrm{~N} & 0.008418 & 2.189825 & 2.091897 \\ \mathrm{~N} & 0.008418 & -2.189825 & 2.091897 \\ \mathrm{~N} & 0.008418 & 2.189825 & -2.091897 \\ \mathrm{~N} & 0.008418 & -2.189825 & -2.091897 \\ \mathrm{~N} & 5.218544 & 2.189825 & -2.091897 \\ \mathrm{~N} & 5.218544 & -2.189825 & -2.091897 \\ \mathrm{~N} & 5.218544 & 2.189825 & 2.091897 \\ \mathrm{~N} & 5.218544 & -2.189825 & 2.091897 \\ \mathrm{C} & 2.613796 & -4.008546 & 3.517023 \\ \mathrm{H} & 1.706799 & -3.555818 & 3.119862 \\ \mathrm{H} & 3.520792 & -3.555818 & 3.119862 \\ \mathrm{Cl} & 2.613792 & -3.782863 & 5.306155 \\ \mathrm{Cl} & 2.613792 & -5.753061 & 3.060301 \\ \mathrm{C} & 2.613796 & 4.008546 & 3.517023 \\ \mathrm{H} & 1.706799 & 3.555872 & 3.119800 \\ \mathrm{H} & 3.520792 & 3.555872 & 3.119800 \\ \mathrm{Cl} & 2.613792 & 5.753123 & 3.060540 \\ \mathrm{Cl} & 2.613792 & 3.782618 & 5.306124 \\ \mathrm{C} & 2.613796 & 4.008546 & -3.517023 \\ \mathrm{H} & 1.706799 & 3.555818 & -3.119862 \\ \mathrm{H} & 3.520792 & 3.555818 & -3.119862 \\ \mathrm{Cl} & 2.613792 & 3.782863 & -5.306155 \\ \mathrm{Cl} & 2.613792 & 5.753061 & -3.060301 \\ \mathrm{C} & 2.613796 & -4.008546 & -3.517023 \\ \mathrm{H} & 1.706799 & -3.555872 & -3.119800 \\ \mathrm{H} & 3.520792 & -3.555872 & -3.119800 \\ \mathrm{Cl} & 2.613792 & -5.753123 & -3.060540 \\ \mathrm{Cl} & 2.613792 & -3.782618 & -5.306124\end{array}$

$E(U B+H F-L Y P)=-4734.11675600$ $\left\langle S^{* *} 2\right\rangle=1.0171$ 


\begin{tabular}{ccccc} 
& 1 & 2 & 3 & \\
Frequencies -- & 8.5695 & 9.2016 & \multicolumn{1}{c}{11.0464} \\
Frequencies -- & 4 & 5 & 6 & \\
& 11.0806 & 14.3082 & & 16.1840
\end{tabular}

Mulliken atomic spin density:

\begin{tabular}{|c|c|}
\hline TCNE$^{-}(1)$ & 0.99 \\
\hline TCNE$^{-}(2)$ & -0.99 \\
\hline $\mathrm{CH}_{2} \mathrm{Cl}_{2}(1)$ & 0.00 \\
\hline $\mathrm{CH}_{2} \mathrm{Cl}_{2}(2)$ & 0.00 \\
\hline $\mathrm{CH}_{2} \mathrm{Cl}_{2}(3)$ & 0.00 \\
\hline $\mathrm{CH}_{2} \mathrm{Cl}_{2}(4)$ & 0.00 \\
\hline
\end{tabular}

Mulliken atomic charges:

$\begin{array}{ll}\text { TCNE}^{-}(1) & -0.93 \\ \text { TCNE- (2) } & --0.93 \\ & \\ \mathrm{CH}_{2} \mathrm{Cl}_{2}(1) & -0.03 \\ \mathrm{CH}_{2} \mathrm{Cl}_{2}(2) & -0.03 \\ \mathrm{CH}_{2} \mathrm{Cl}_{2}(3) & -0.03 \\ \mathrm{CH}_{2} \mathrm{Cl}_{2}(4) & -0.03\end{array}$




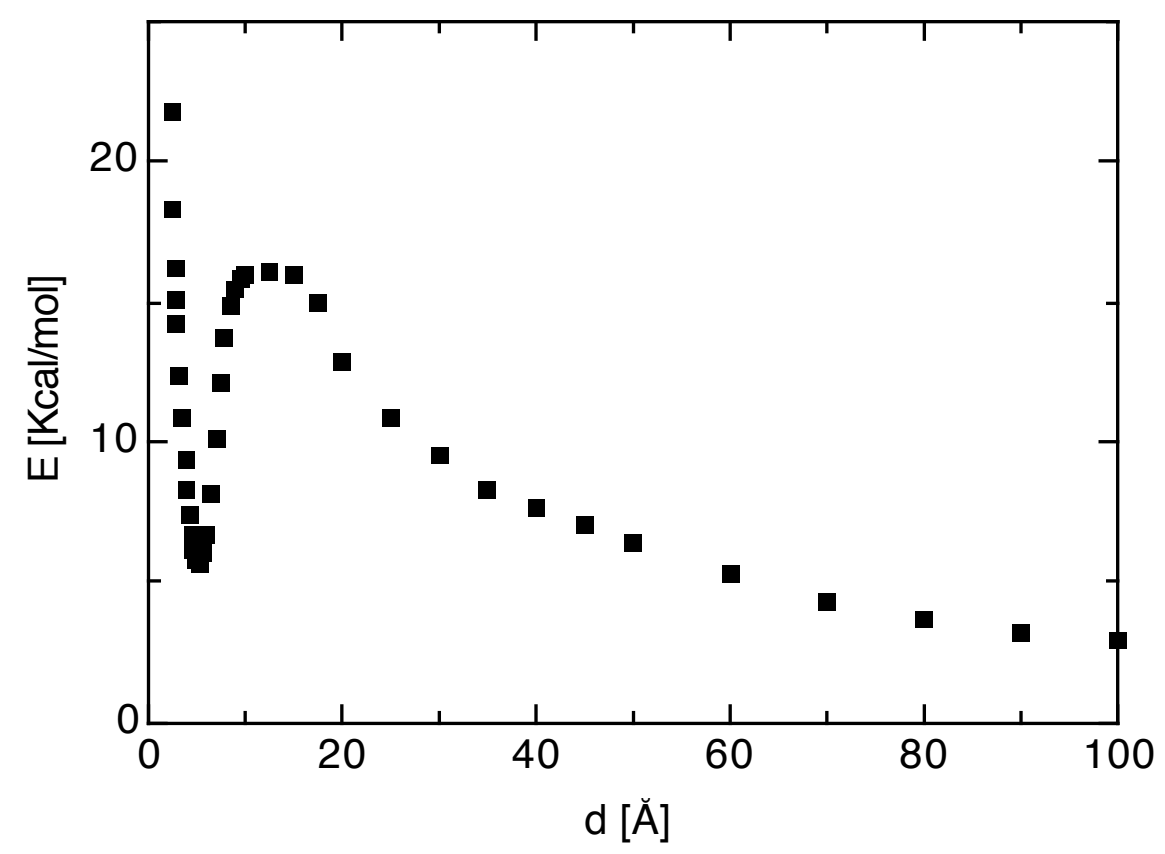

Figure S1. Variation of the interaction energy $(\mathrm{E})$ of the $[\mathrm{TCNE}]_{2}{ }^{2-}\left(\mathrm{CH}_{2} \mathrm{Cl}_{2}\right)_{4}$ aggregate when the distance between the two anions (d) is increased, whole leaving untouched the central $\left(\mathrm{CH}_{2} \mathrm{Cl}_{2}\right)_{4}$ aggregate. The calculation has been computed for the open-shell singlet at the UB3LYP/6-31+G(d) level. 
(a) Open-shell singlet
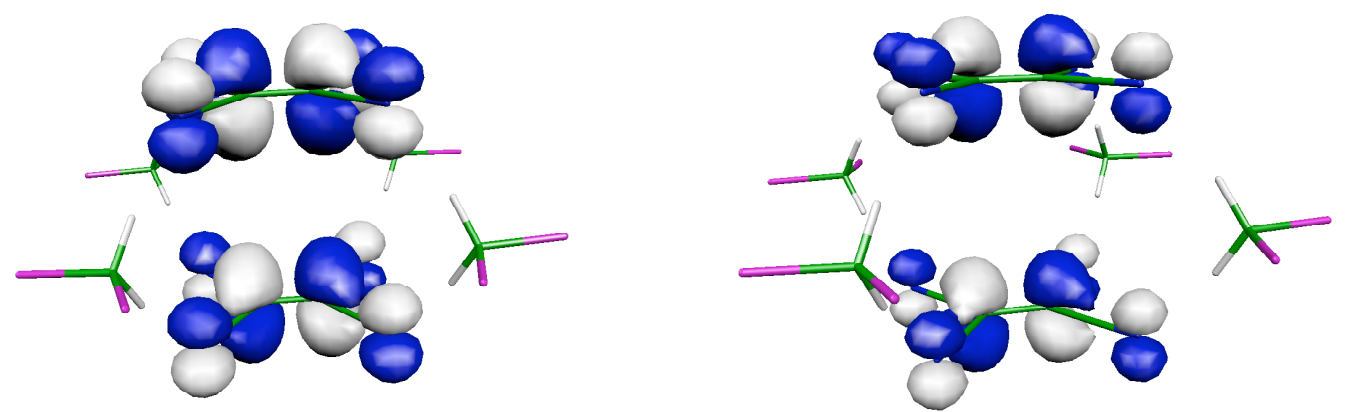

(b) Closed-shell singlet
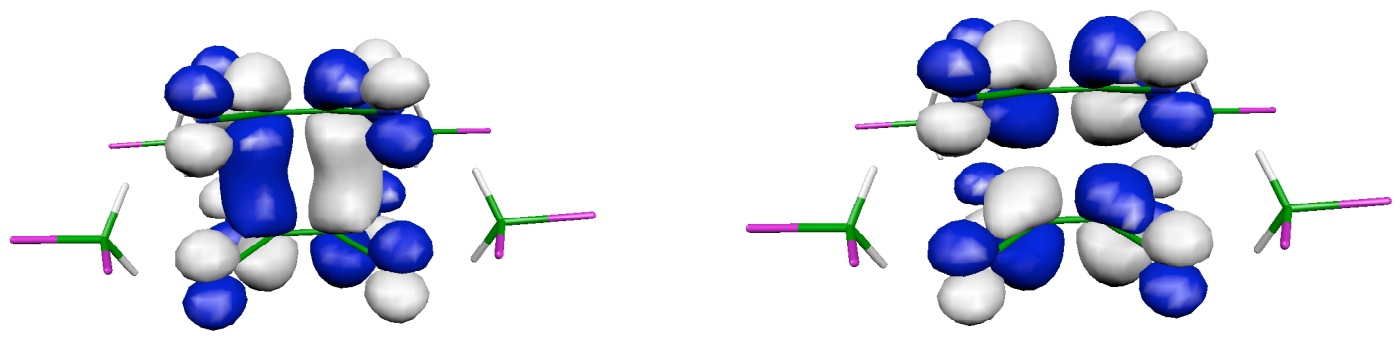

(c) Triplet
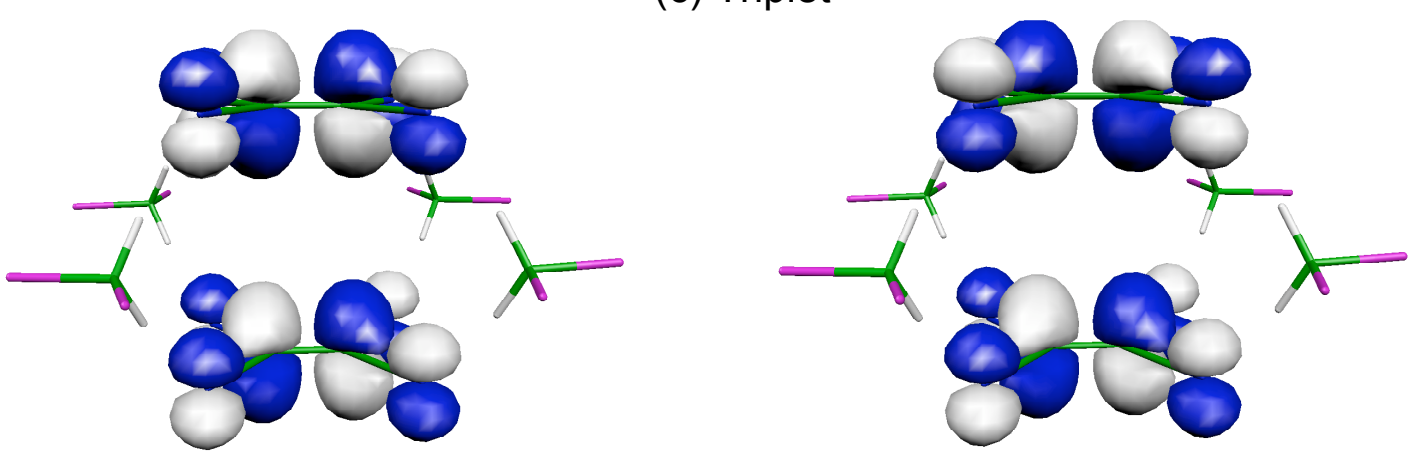

Figure S2. (a): Shape of the two SOMO of the open-shell singlet state; (b) shape of the HOMO and LUMO of the closed-shell singlet; (c) shape of the two SOMO orbitals of the triplet state. 